\title{
A Good Leaf Order on Simplicial Trees
}

\author{
Sara Faridi*
}

September 17, 2021

\begin{abstract}
Using the existence of a good leaf in every simplicial tree, we order the facets of a simplicial tree in order to find combinatorial information about the Betti numbers of its facet ideal. Applications include an Eliahou-Kervaire splitting of the ideal, as well as a refinement of a recursive formula of Hà and Van Tuyl for computing the graded Betti numbers of simplicial trees.
\end{abstract}

Dedicated to Tony Geramita for his many contributions to Mathematics

\section{Introduction}

Given a monomial ideal $I$ in a polynomial ring $R=k\left[x_{1}, \ldots, x_{n}\right]$ over a field $k$, a minimal free resolution of $I$ is an exact sequence of free $R$-modules

$$
0 \rightarrow \bigoplus_{d} R(-d)^{\beta_{p, d}} \rightarrow \cdots \rightarrow \bigoplus_{d} R(-d)^{\beta_{0, d}} \rightarrow I \rightarrow 0
$$

of $R / I$ in which $R(-d)$ denotes the graded free module obtained by shifting the degrees of elements in $R$ by $d$. The numbers $\beta_{i, d}$, which we shall refer to as the $i$-th $\mathbb{N}$-graded Betti numbers of degree $d$ of $R / I$, are independent of the choice of graded minimal finite free resolution.

Questions about Betti numbers - including when they vanish and when they do not, what bounds they have, how they relate to the base field $k$ and what are the most effective ways to compute them - are of particular interest in combinatorial commutative algebra. Via a method called polarization $[\overline{F r}]$, it turns out that it is enough to consider such questions for square-free monomial ideals [GPW]; i.e. a monomial ideal in which the generators are square-free monomials.

To a square-free monomial ideal $I$ one can associate a unique simplicial complex called its facet complex. Conversely, every simplicial complex has a unique monomial ideal assigned to it called its facet ideal [F1]. Simplicial trees [F1] and related structures were developed as a class of simplicial complexes that generalize graph-trees, so that their facet ideals have similar properties to those of edge ideals of graphs discovered in a series of works by Villarreal and his coauthors [V].

\footnotetext{
${ }^{*}$ Department of Mathematics and Statistics, Dalhousie University, Halifax, Canada, faridi@ mathstat.dal.ca. Research supported by NSERC. The author acknowledges the hospitality of MSRI in Berkeley, CA where part of this work was completed.
} 
This paper offers an order on the monomials generating the facet ideal of a simplicial tree which uses the existence of a "good leaf" in every simplicial tree [HHTZ]. This order in itself is combinatorially interesting and useful, but it turns out that it also produces a "splitting" [EK] of the facet ideal of a tree which gives bounds on the Betti numbers of the ideal.

Our good leaf order also makes it possible to refine a recursive formula of Hà and Van Tuyl [HV] for computing Betti numbers of facet ideals of simplicial trees, and to apply it to classes of trees with strict good leaf orders. The idea here is that a good leaf order will split an ideal to some extent, and within each one of these split pieces, one can apply Hà and Van Tuyl's formula quite efficiently if the order is strict.

\section{Simplicial complexes, trees and forests}

Definition 2.1 (simplicial complexes). A simplicial complex $\Delta$ over a set of vertices $V(\Delta)=$ $\left\{v_{1}, \ldots, v_{n}\right\}$ is a collection of subsets of $V(\Delta)$, with the property that $\left\{v_{i}\right\} \in \Delta$ for all $i$, and if $F \in \Delta$ then all subsets of $F$ are also in $\Delta$. An element of $\Delta$ is called a face of $\Delta$, and the dimension of a face $F$ of $\Delta$ is defined as $|F|-1$, where $|F|$ is the number of vertices of $F$. The faces of dimensions 0 and 1 are called vertices and edges, respectively, and $\operatorname{dim} \emptyset=-1$. The maximal faces of $\Delta$ under inclusion are called facets of $\Delta$. The dimension of the simplicial complex $\Delta$ is the maximal dimension of its facets. A subcollection of $\Delta$ is a simplicial complex whose facets are also facets of $\Delta$.

A simplicial complex $\Delta$ is connected if for every pair of facets $F, G$ of $\Delta$, there exists a sequence of facets $F_{1}, \ldots, F_{r}$ of $\Delta$ such that $F_{1}=F, F_{r}=G$ and $F_{s} \cap F_{s+1} \neq \emptyset$ for $1 \leqslant s<r$.

We use the notation $\left\langle F_{1}, \ldots, F_{q}\right\rangle$ to denote the simplicial complex with facets $F_{1}, \ldots, F_{q}$, and we call it the simplicial complex generated by $F_{1}, \ldots, F_{q}$. By removing the facet $F_{i}$ from $\Delta$ we mean the simplicial complex $\Delta \backslash\left\langle F_{i}\right\rangle$ which is generated by $\left\{F_{1}, \ldots, F_{q}\right\} \backslash\left\{F_{i}\right\}$.

Definition 2.2 (Leaf, joint, simplicial trees and forests [F1]). A facet $F$ of a simplicial complex $\Delta$ is called a leaf if either $F$ is the only facet of $\Delta$, or $F$ intersects $\Delta \backslash\langle F\rangle$ in a face of $\Delta \backslash\langle F\rangle$. If $F$ is a leaf and $\Delta$ has more than one facet, then for some facet $G \in \Delta \backslash\langle F\rangle$ we have $F \cap H \subseteq G$ for all $H \in \Delta \backslash\langle F\rangle$. Such a facet $G$ is called a joint of $F$.

A simplicial complex $\Delta$ is a simplicial forest if every nonempty subcollection of $\Delta$ has a leaf. A connected simplicial forest is called a simplicial tree.

It follows easily from the definition that a leaf must always contain at least one free vertex, that is a vertex that belongs to no other facet of $\Delta$.

Example 2.3. The facets $F_{0}, F_{2}$ and $F_{4}$ are all leaves of the simplicial tree in Figure 1 The first two have $F_{1}$ as a joint and $F_{4}$ has $F_{3}$ as a joint.

Definition 2.4 (Good leaf [Z, CFS]). A facet $F$ of a simplicial complex $\Delta$ is called a good leaf of $\Delta$ if $F$ is a leaf of every subcollection of $\Delta$ which contains $F$. 


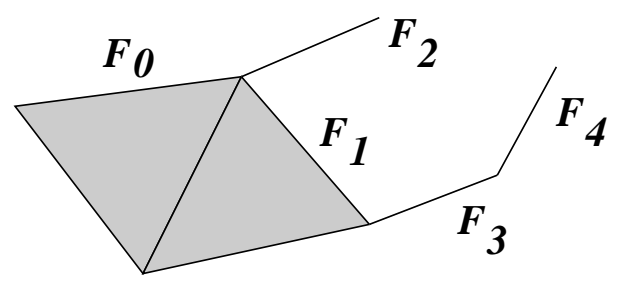

Figure 1: Good leaves

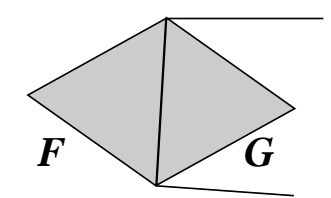

Figure 2: A leaf that is not a good leaf

All leaves of the simplicial tree in Figure 1 are good leaves. Figure 2 contains an example of a leaf $F$ in a simplicial tree which is not a good leaf: if we remove the facet $G$ then $F$ is no longer a leaf.

Good leaves were studied in $[\bar{Z}]$ and then independently in [CFS] (where they were called "reducible leaves"). In both sources the existence of such a leaf in every tree was conjectured but not proved; the proof came later, using incidence matrices.

Theorem 2.5 ([[HHTZ]). Every simplicial tree contains a good leaf.

Definition 2.6 (Facet ideal, facet complex [F1]). Let $\Delta$ be a simplicial complex with vertex set $\left\{x_{1}, \ldots, x_{n}\right\}$, and let $R=k\left[x_{1}, \ldots, x_{n}\right]$ be a polynomial ring over a field $k$ with variables corresponding to the vertices of $\Delta$. The facet ideal of $\Delta$, denoted by $\mathcal{F}(\Delta)$, is an ideal of $R$ whose generators are monomials, each of which is the products of the variables labeling the vertices of a facet of $\Delta$. Given a square-free monomial ideal $I$ in $R$, the facet complex of $I$ is the simplicial complex whose facets are the set of variables appearing in each monomial generator of $I$.

Example 2.7. If $I=(x y, y z u, x z)$ is a monomial ideal in $R=k[x, y, z, u]$, its facet complex is the simplicial complex $\Delta=\langle\{x, y\},\{y, z, u\},\{x, z\}\rangle$. Similarly $I$ is the facet ideal of $\Delta$.

It is clear from the definition and example that every square-free monomial ideal has a unique facet complex, and every simplicial complex has a unique facet ideal. Because of this one-toone correspondence we often abuse notation and use facets and monomials interchangeably. For example we say $F \cup G=\operatorname{lcm}(F, G)$ to imply the union of two facets $F$ and $G$ or the least common multiple of two monomials [corresponding to the facets] $F$ and $G$.

Trees behave well under localization:

Lemma 2.8 (Localization of a tree is a forest [F1]). Let $\Delta$ be a simplicial tree with vertices $x_{1}, \ldots, x_{n}$, and let $I$ be the facet ideal of $\Delta$ in the polynomial ring $R=k\left[x_{1}, \ldots, x_{n}\right]$ where $k$ is a field. Then for any prime ideal $p$ of $R, I_{p}$ is the facet ideal of a simplicial forest. 
For a simplicial complex $\Delta$ with a facet $F$, we use the notation $\Delta_{\bar{F}}$ for facet complex of the localization $\mathcal{F}(\Delta)$ at the ideal generated by the the complement of the facet $F$.

\section{Good leaf orders}

From its definition it is immediate that a good leaf $F_{0}$ of a tree $\Delta$ induces an order $F_{0}, F_{1}, \ldots, F_{q}$ on the facets of $\Delta$ so that

$$
F_{0} \cap F_{1} \supseteq F_{0} \cap F_{2} \supseteq \cdots \supseteq F_{0} \cap F_{q} .
$$

Our goal in this section is to demonstrate that this order can be refined so that $\Delta$ is built leaf by leaf starting from the good leaf $F_{0}$. In other words, the order can be written so that for $i \leqslant q, F_{i}$ is a leaf of $\Delta_{i}=\left\langle F_{0}, \ldots, F_{i}\right\rangle$. Such an order on the facets of $\Delta$ will be called a good leaf order on $\Delta$.

Example 3.1. Let $\Delta$ be the simplicial tree in Figure 1 Then $F_{0}$ is a good leaf and the labeling of facets $F_{0}, \ldots, F_{4}$ is a good leaf order on $\Delta$, since $F_{0} \cap F_{1} \supseteq \cdots \supseteq F_{0} \cap F_{4}$. Note that even though $F_{0} \cap F_{1} \supseteq F_{0} \cap F_{2} \supseteq F_{0} \cap F_{4} \supseteq F_{0} \cap F_{3}$, this latter order $F_{0}, F_{1}, F_{2}, F_{4} . F_{3}$ is not a good leaf order since $F_{3}$ is not a leaf of $\Delta$.

We show that every simplicial tree (forest) has a good leaf order.

Lemma 3.2. Suppose $\Delta=\langle F, G, H\rangle$ is a simplicial tree with $F \cap G \not H H$ and $F \cap H \nsubseteq G$. Then $G$ and $H$ are the leaves of the tree $\Delta$ and $F$ is the common joint so that $G \cap H \subseteq F$.

Proof. If $F$ is a leaf, then either $F \cap G \subseteq H$ or $F \cap H \subseteq G$. Either case is a contradiction, so the two leaves of the tree have to be $G$ and $H$. If $H$ is a joint of the leaf $G$ then $F \cap G \subseteq H$ which is again a contradiction, so $F$ is the joint of $G$. Similarly, $F$ is the joint of $H$, and we have $G \cap H \subseteq F$.

Proposition 3.3 (First step to build good leaf order). Let $\Delta$ be a simplicial tree with a good leaf $F_{0}$ and good leaf order

$$
F_{0} \cap F_{1} \supseteq F_{0} \cap F_{2} \supseteq \cdots \supseteq F_{0} \cap F_{q} .
$$

Let $1 \leqslant a \leqslant q$ and $0 \leqslant b<a$ and

$$
F_{0} \cap F_{a-b-1} \supsetneq F_{0} \cap F_{a-b}=\cdots=F_{0} \cap F_{a} .
$$

Then one of $F_{a-b}, \ldots, F_{a}$ is a leaf of $\left\langle F_{0}, \ldots, F_{a}\right\rangle$.

Proof. Let $\Gamma=\left\langle F_{0}, \ldots, F_{a}\right\rangle$. The subcollection $\Omega=\left\langle F_{0}, \ldots, F_{a-b-1}\right\rangle$ of $\Gamma$ is connected as all facets have nonempty intersection with $F_{0}$. If $\Gamma$ is disconnected, $\Omega$ will be contained in one of the connected components of $\Gamma$, and there will be another connected component $\Sigma$ whose facets are from $F_{a-b}, \ldots, F_{a}$. Since $\Sigma$ is a subcollection of a tree, it must have a leaf, and that leaf will be a leaf of $\Gamma$ as well. So one of $F_{a-b}, \ldots, F_{a}$ will be a leaf of $\Gamma$.

We now assume that $\Gamma$ is connected and proceed by induction on $a$ to prove our claim. If $a=1$ then clearly $F_{1}$ is a leaf of the tree $\Gamma=\left\langle F_{0}, F_{1}\right\rangle$. If $a=2$ then since $F_{2} \cap F_{0} \subset F_{1}$, the facet $F_{2}$ must be a leaf with joint $F_{1}$. 
Now suppose that $a>2$ and the statement is true up to the $(a-1)$-st step. If $a-b=1$ then

$$
F_{0} \cap F_{1}=F_{0} \cap F_{2}=\cdots=F_{0} \cap F_{a} .
$$

By [F2] Lemma 4.1 we know that $\Gamma$ must have two leaves, and so one of the facets $F_{1}, \ldots, F_{a}$ is a leaf.

We assume that $a-b \geqslant 2$ and neither one of $F_{a}, \ldots, F_{a-b}$ is a leaf of $\Gamma$. There are two possible cases.

1. The case $b=0$. Then $F_{0} \cap F_{a-1} \supsetneq F_{0} \cap F_{a}$. If $\Gamma^{\prime}=\left\langle F_{0}, \ldots, F_{a-2}, F_{a}\right\rangle$ then are two scenarios.

(a) If $\Gamma^{\prime}$ is disconnected, then the facet $F_{a}$ alone is a connected component of $\Gamma^{\prime}$ (since all other facets intersect $F_{0}$ ) and therefore $F_{a}$ is a leaf of $\Gamma^{\prime}$ and $F_{a} \cap F_{i}=\emptyset$ for $i=0, \ldots, a-2$. Since $\Gamma$ is connected, $F_{a-1} \cap F_{a} \neq \emptyset$, and therefore $F_{a}$ is a leaf of $\Gamma$ with joint $F_{a-1}$.

(b) If $\Gamma^{\prime}$ is connected, we apply the induction hypothesis to the tree $\Gamma^{\prime}$ with good leaf $F_{0}$. In the ordering of the facets of $\Gamma^{\prime}, F_{a}$ can only be at the right end of the sequence (since $F_{0} \cap F_{a-2} \supsetneq F_{0} \cap F_{a}$ ). So $F_{a}$ is a leaf of $\Gamma^{\prime}$ and hence there is a joint $F_{j} \in$ $\left\{F_{0}, \ldots, F_{a-2}\right\}$ such that $F_{a} \cap F_{k} \subseteq F_{j}$ for all $F_{k} \in\left\{F_{0}, \ldots, F_{a-2}\right\}$.

If $F_{a}$ is not a leaf of $\Gamma$ then $F_{a} \cap F_{a-1} \nsubseteq F_{j}$. It also follows that $F_{a} \cap F_{j} \nsubseteq F_{a-1}$, as otherwise $F_{a-1}$ would be a joint of $F_{a}$. Therefore, we can now apply Lemma 3.2 to the tree $\left\langle F_{j}, F_{a-1}, F_{a}\right\rangle$ to conclude that $F_{j} \cap F_{a-1} \subseteq F_{a}$. It follows that

$$
F_{0} \cap F_{j} \cap F_{a-1} \subseteq F_{0} \cap F_{a} \Longrightarrow F_{0} \cap F_{a-1} \subseteq F_{0} \cap F_{a} \subsetneq F_{a-1} \cap F_{0}
$$

which is a contradiction. So $F_{a}$ has to be a leaf of $\Gamma$ and we are done.

2. The case $b>0$. We keep the good leaf $F_{0}$ and generate complexes $\Gamma_{i}=\Gamma \backslash\left\langle F_{i}\right\rangle$ for $i \in$ $\{1, \ldots, a\}$. By induction hypothesis each $\Gamma_{i}$ has a leaf $F_{u_{i}}$ where $u_{i} \in\{a-b, \ldots, \hat{i}, \ldots, a\}$. Since there are a total of $b+1$ facets that can be leaves of the $\Gamma_{i}$, and there are $a>b+1$ of the complexes $\Gamma_{i}$ (recall that we are assuming $a-b \geqslant 2$ ), we must have $u_{i}=u_{j}=u$ for some distinct $i, j \in\{1, \ldots, a\}$. Suppose $F_{v_{i}}$ and $F_{v_{j}}$ are the joints of $F_{u}$ in $\Gamma_{i}$ and $\Gamma_{j}$, respectively. So we have

$$
\begin{aligned}
& F_{u} \cap F_{h} \subseteq F_{v_{i}} \text { if } h \neq i \\
& F_{u} \cap F_{h} \subseteq F_{v_{j}} \text { if } h \neq j .
\end{aligned}
$$

These two embeddings imply that

$$
\begin{aligned}
& F_{u} \cap F_{j} \subseteq F_{v_{i}} \cap F_{u} \subseteq F_{v_{j}} \text { if } v_{i} \neq j \\
& F_{u} \cap F_{i} \subseteq F_{v_{j}} \cap F_{u} \subseteq F_{v_{i}} \text { if } v_{j} \neq i .
\end{aligned}
$$


Suppose $v_{i} \neq j$. Then from (1) and (2) we can see that $F_{u}$ is a leaf of $\Gamma$ with joint $F_{v_{j}}$. Similarly $F_{u}$ is a leaf of $\Gamma$ if $v_{j} \neq i$. So $F_{u}$ is a leaf of $\Gamma$ unless $v_{i}=j$ and $v_{j}=i$ are the only possible joints for $F_{u}$ in $\Gamma_{i}$ and $\Gamma_{j}$, respectively. In this case (1) turns into

$$
\begin{aligned}
& F_{u} \cap F_{h} \subseteq F_{j} \text { if } h \neq i \\
& F_{u} \cap F_{h} \subseteq F_{i} \text { if } h \neq j .
\end{aligned}
$$

Now consider $\Gamma_{u}=\Delta \backslash\left\langle F_{u}\right\rangle$, which by induction hypothesis must have a leaf $F_{v}$ with $v \in\{a-b, \ldots, a\} \backslash\{u\}$ and a joint $F_{t}$. Since $F_{i}, F_{j} \in \Gamma_{u}$, we must have

$$
\begin{aligned}
& F_{i} \cap F_{v} \subseteq F_{t} \text { if } v \neq i \\
& F_{j} \cap F_{v} \subseteq F_{t} \text { if } v \neq j .
\end{aligned}
$$

Once again, we consider two cases.

(a) If $v$ can be selected outside $\{i, j\}$, we combine (4) with (3) to get

$$
F_{u} \cap F_{v} \subseteq F_{j} \cap F_{v} \subseteq F_{t}
$$

meaning that $F_{v}$ is a leaf of $\Gamma$.

(b) If $v$ must be in $\{i, j\}$, then the only leaves of $\Gamma_{u}$ are $F_{i}$ and $F_{j}$. As $F_{0} \in \Gamma_{u}$ is a good leaf of $\Delta$, one of $i$ and $j$ must be 0 , say $j=0$. But now we have

$$
F_{u} \cap F_{j}=F_{u} \cap F_{0} \subseteq F_{i}
$$

which together with (3) implies that $F_{u}$ is a leaf of $\Gamma$ with joint $F_{i}$.

Our main theorem is now just a direct consequence of Proposition 3.3, with a bit more added to it.

Theorem 3.4 (Main theorem: good leaf orders). Let $\Delta$ be a simplicial tree with a good leaf $F_{0}$. Then there is an order $F_{0}, F_{1}, \ldots, F_{q}$ on the facets of $\Delta$ such that

1. $F_{0} \cap F_{1} \supseteq F_{0} \cap F_{2} \supseteq \cdots \supseteq F_{0} \cap F_{q}$, and

2. The facet $F_{i}$ is a leaf of $\Delta_{i}=\left\langle F_{0}, \ldots, F_{i}\right\rangle$ for $0 \leqslant i \leqslant q$.

3. The facet $F_{i-1}$ is a either a leaf of $\Delta_{i}$ with the same joint as it has in $\Delta_{i-1}$, or it is the unique joint of $F_{i}$ in $\Delta_{i}$, for $1 \leqslant i \leqslant q$.

4. $\Delta_{i}=\left\langle F_{0}, \ldots, F_{i}\right\rangle$ is connected for $0 \leqslant i \leqslant q$. 
Proof. The good leaf $F_{0}$ induces an order on the facets of $\Delta$ that satisfies the first property. We need to refine this order to achieve the second property. Let $i \in\{1, \ldots, q\}$. Starting from the beginning, here is how we proceed. For $i \in\{1, \ldots, q\}$ let $c_{i}$ be the largest nonnegative integer such that $F_{i} \cap F_{0}=F_{i+c_{i}} \cap F_{0}$ where $i+c_{i} \leqslant q$.

Set $i=1$.

Step 1 If $c_{i}=0$ then set $i:=i+1$ and go back to Step 1 .

Step 2 If $c_{i}>0$ then we reorder $F_{i}, \ldots, F_{i+c_{i}}$ as follows. By Proposition 3.3 there is a leaf $F_{\ell_{c_{i}}} \in$ $\left\{F_{i}, \ldots, F_{i+c_{i}}\right\}$ of $\Gamma=\left\langle F_{0}, \ldots, F_{i+c_{i}}\right\rangle$. Applying the same proposition again there is a leaf $F_{\ell_{c_{i}-1}} \in\left\{F_{i}, \ldots, F_{i+c_{i}}\right\} \backslash\left\{F_{\ell_{c_{i}}}\right\}$ of $\Gamma \backslash\left\langle F_{\ell_{c_{i}}}\right\rangle$. We continue this way $c_{i}+1$ times and in the end we have a sequence

$$
F_{\ell_{0}}, F_{\ell_{1}}, \ldots, F_{\ell_{i}}
$$

which is a reordering of the facets $F_{i}, \ldots, F_{i+c_{i}}$ that satisfies both properties (1) and (2) in the statement of the theorem. We relabel $F_{i}, \ldots, F_{i+c_{i}}$ with this new order and set $i:=i+c_{i}+1$.

Step 3 If $i>q$ we stop and otherwise we go back to Step 1.

At the end of this algorithm, the facets of $\Delta$ have the desired order.

To prove the third part of the theorem, note that as $F_{i-1}$ is a leaf in $\Delta_{i-1}$, it has a set of free vertices in $\Delta_{i-1}$ which we call $A$. There are two scenarios.

- If $F_{i} \cap A \neq \emptyset$, then $F_{i-1}$ has to be the unique joint of $F_{i}$ in $\Delta_{i}$, as no other facet of $\Delta_{i}$ would contain any element of $A$.

- If $F_{i} \cap A=\emptyset$, then $F_{i} \cap F_{i-1} \subseteq \Delta_{i-2} \cap F_{i-1} \subseteq F_{\alpha}$, where $F_{\alpha}$ is the joint of $F_{i-1}$ in $\Delta_{i-1}$. Therefore, $F_{i-1}$ is a leaf of $\Delta_{i}$.

Finally to see that $\Delta_{i}$ is connected for every $i$, we consider two situations.

1. $F_{i} \cap F_{0} \neq \emptyset$. In this case $\Delta_{i}$ is connected as all facets of $\Delta_{i}$ intersect $F_{0}$.

2. $F_{i} \cap F_{0}=\emptyset$. If $i=q$ then $\Delta_{i}=\Delta$ which is connected. Now we assume that $i$ is the smallest index with $F_{i} \cap F_{0}=\emptyset$, and $c_{i}>0$, and we consider how $\Delta_{i}, \ldots, \Delta_{q}=\Delta$ are built in Step 2. We start from $\Delta$, and pick a leaf for $\Delta$ from $F_{i}, \ldots, F_{q}$. We call this facet $F_{q}$ and we know already that $\Delta_{q}=\Delta$ must be connected. To pick $\Delta_{q-1}$ we remove the leaf $F_{q}$ from $\Delta$, and so $\Delta_{q-1}$ has to be connected. To build $\Delta_{q-2}$ we again remove a leaf from $\Delta_{q-1}$, which forces $\Delta_{q-2}$ to be connected, and so on until we reach $\Delta_{i}$, which by the same reasoning has to be connected. 


\section{The effect of good leaf orders on resolutions}

Recall that for a monomial ideal $I$, the notation $\mathcal{G}(I)$ denotes the unique minimal monomial generating set for $I$.

Definition 4.1 (Splitting [EK]). A monomial ideal $I$ is called splittable if one can write $I=J+K$ for two nonzero monomial ideals $J$ and $K$, such that

1. $\mathcal{G}(I)$ is the disjoint union of $\mathcal{G}(J)$ and $\mathcal{G}(K)$;

2. There is a splitting function $\mathcal{G}(J \cap K) \rightarrow \mathcal{G}(J) \times \mathcal{G}(K)$ taking each $w \in \mathcal{G}(J \cap K)$ to $(\phi(w), \psi(w))$ satisfying

(a) For each $w \in \mathcal{G}(J \cap K), w=\operatorname{lcm}(\phi(w), \psi(w))$

(b) For each $S \subseteq \mathcal{G}(J \cap K)$, $\operatorname{lcm}(\phi(S))$ and $\operatorname{lcm}(\psi(S))$ strictly divide $\operatorname{lcm}(S)$.

If a monomial ideal is splittable, then its Betti numbers can be broken down into those of subideals.

Theorem $4.2([\overline{\mathrm{EK}}, \mid \mathrm{Fa}])$. If I is a monomial ideal with a splitting $I=J+K$, then for all $i, j \geqslant 0$

$$
\beta_{i, j}(I)=\beta_{i, j}(J)+\beta_{i, j}(K)+\beta_{i-1, j}(J \cap K) .
$$

Our next observation is that a good leaf order on a simplicial tree provides a basic splitting of its facet ideal.

Theorem 4.3 (Splitting using a good leaf order). If I is the facet ideal of a simplicial tree $\Delta$ with a good leaf $F_{0}$ and good leaf order

$$
F_{0} \cap F_{1} \supseteq F_{0} \cap F_{2} \supseteq \ldots \supseteq F_{0} \cap F_{t} \supsetneq F_{0} \cap F_{t+1}=\ldots=F_{0} \cap F_{q}=\emptyset
$$

and $J=\left(F_{0}, \ldots, F_{t}\right)$ and $K=\left(F_{t+1}, \ldots, F_{q}\right)$, then $I=J+K$ is a splitting of $I$.

Proof. It is clear that $I=J+K$. We number the vertices of $F_{0}, \ldots, F_{t}$ in some order as $x_{1}, \ldots, x_{m}$. We will build $\phi$ and $\psi$ as in Definition 4.1. Suppose $L \in \mathcal{G}(J \cap K)$. Then there are facets $F_{i}$ and $F_{j}$ such that $i \leqslant t<j$ such that $L=\operatorname{lcm}\left(F_{i}, F_{j}\right)$. Of all choices of such $F_{i}$ we pick one minimal with respect to lex order and call it $G_{L}$, and there is only one choice for $F_{j}$ (since each $F_{j}$ adds one or more new vertices to the sequence $\left.F_{0}, \ldots, F_{j-1}\right)$; call this facet $H_{L}$. So we have $L=\operatorname{lcm}\left(G_{L}, H_{L}\right)$. Let $\phi(L)=G_{L}$ and $\psi(L)=H_{L}$ so that we have a map

$$
\begin{array}{cl}
\mathcal{G}(J \cap K) & \rightarrow \mathcal{G}(J) \times \mathcal{G}(K) \\
L & \rightarrow(\phi(L), \psi(L))=\left(G_{L}, H_{L}\right)
\end{array}
$$

We only need to show that Condition (b) in Definition 4.1 holds. Suppose $S=\left\{L_{1}, \ldots, L_{r}\right\} \subseteq$ $\mathcal{G}(J \cap K)$. Suppose, as before, for each $i$ we can write $L_{i}=\operatorname{lcm}\left(G_{L_{i}}, H_{L_{i}}\right)=G_{L_{i}} \cup H_{L_{i}}$ where $G_{L_{i}} \in \mathcal{G}(K)$ and $H_{L_{i}} \in \mathcal{G}(K)$. We need to show 
1. $G_{L_{1}} \cup \cdots \cup G_{L_{r}} \subsetneq L_{1} \cup \cdots \cup L_{r}$.

This is clear since each of the $L_{i}$ contains vertices that are in $\mathcal{G}(K)$ but not in $\mathcal{G}(J)$.

2. $H_{L_{1}} \cup \cdots \cup H_{L_{r}} \subsetneq L_{1} \cup \cdots \cup L_{r}$

Each of the $L_{i}$ has a nonempty intersection with $F_{0}$, but $H_{L_{i}} \cap F_{0}=\emptyset$, which makes the inclusion above strict.

So we have shown that we have a splitting which completes the proof.

As a result, we can use good leaf orders to bound invariants related to resolutions of trees. Recall that the regularity of an ideal $I$, denoted by $\operatorname{reg}(I)$, is the maximum value of $j-i$ where $\beta_{i, j}(I) \neq 0$. The projective dimension of $I$, denoted by $\operatorname{proj\operatorname {dim}}(I)$, is the maximum value of $i$ where $\beta_{i, j}(I) \neq 0$ for some $j$. The projective dimension and regularity measure the "length" and the "width" of a minimal free resolution, as can be seen in the Betti diagram of the ideal; see Example 4.5 below. For a simplicial complex $\Gamma$ we often use the notation $\beta_{i, j}(\Gamma), \operatorname{reg}(\Gamma)$ and $\operatorname{projdim}(\Gamma)$ to indicate the Betti numbers, regularity and projective dimension of $\mathcal{F}(\Gamma)$.

The following statement is a direct application of theorems 4.2 and 4.3 .

Corollary 4.4 (Bounds on Betti numbers of trees). Suppose $\Delta$ is a simplicial tree that can be partitioned into subcollections $\Delta_{0}, \ldots, \Delta_{s}$, each of which is a tree, and such that for each $i=$ $0, \ldots, s$, setting $a_{0}=0$ we have:

1. $\Delta_{i}=\left\langle F_{a_{i}}, F_{a_{i}+1}, \ldots, F_{a_{i+1}-1}\right\rangle$ with good leaf $F_{a_{i}}$.

2. $F_{a_{i}+1} \cap F_{a_{i}} \supseteq \ldots \supseteq F_{a_{i+1}-1} \cap F_{a_{i}} \neq \emptyset$ is a good leaf order on $\Delta_{i}$;

3. $F_{a_{i}} \cap F_{j}=\emptyset$ for $j \geqslant a_{i+1}$.

Then

$$
\beta_{i, j}(\Delta) \geqslant \beta_{i, j}\left(\Delta_{0}\right)+\cdots+\beta_{i, j}\left(\Delta_{s}\right)
$$

In particular

$$
\operatorname{projdim}(\Delta) \geqslant \max \left\{\operatorname{projdim}\left(\Delta_{0}\right), \ldots, \operatorname{projdim}\left(\Delta_{s}\right)\right\}
$$

and

$$
\operatorname{reg}(\Delta) \geqslant \max \left\{\operatorname{reg}\left(\Delta_{0}\right), \ldots, \operatorname{reg}\left(\Delta_{s}\right)\right\}
$$

We demonstrate the effect via the example of Figure 1 which we will label below.

Example 4.5. For the ideal $I=(x y z, y z v, y u, v w, w t)$ the facet complex $\Delta$ is

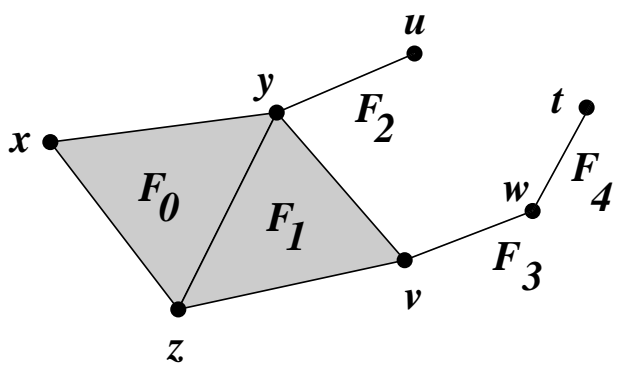


Following the statement of the corollary, we can find a splitting for $I$ by partitioning the facets of $\Delta$ into two trees with the written good leaf orders

$$
\left\langle F_{0}, F_{1}, F_{2}\right\rangle \quad \text { and } \quad\left\langle F_{3}, F_{4}\right\rangle
$$

which correspond, respectively, to the two ideals

$$
J=(x y z, y z v, y u) \quad \text { and } \quad K=(v w, w t) .
$$

We copy the Betti diagrams of $I, J$ and $K$ (in that order) using Macaulay2 [M2].

\begin{tabular}{|c|llll|}
\hline $\mathbf{I}$ & 0 & 1 & 2 & 3 \\
\hline 1 & 3 & 1 &. &. \\
2 & 2 & 6 & 3 &. \\
3 &. & 1 & 2 & 1 \\
\hline
\end{tabular}

\begin{tabular}{|l|lll|}
\hline $\mathbf{J}$ & 0 & 1 & 2 \\
\hline 1 & 1 & $\cdot$ & $\cdot$ \\
2 & 2 & 3 & 1 \\
\hline
\end{tabular}

\begin{tabular}{|r|ll|}
\hline $\mathbf{K}$ & 0 & 1 \\
\hline 1 & 2 & 1 \\
\hline
\end{tabular}

The bounds presented in Corollary 4.4 are now evident from the Betti diagrams:

$$
\operatorname{reg}(I)=3 \geqslant \max \{\operatorname{reg}(J), \operatorname{reg}(K)\}=\max \{2,1\}=2
$$

and

$$
\operatorname{projdim}(I)=3 \geqslant \max \{\operatorname{projdim}(J), \operatorname{projdim}(K)\}=\max \{2,1\}=2
$$

There can be different good leaf orders on a simplicial tree. It would be interesting to know which one gives a "better" splitting, and better bounds for the resolution invariants.

\subsection{Recursive calculations of Betti numbers}

In [HV] Hà and Van Tuyl used Eliahou-Kervaire splittings to reduce the computation of the Betti numbers of a given simplicial forest to that of smaller ones. Our goal here is to show that their formula can be refined in certain cases and be used to compute the Betti numbers of a given simplicial tree in terms of intersections of the faces. The method used is essentially a repeated application of a splitting formula due to Hà and Van Tuyl [ $[\mathrm{HV}]$ to a good leaf order on a given tree, along with an argument that, at every stage, we know what the next splitting to consider should be.

Definition 4.6 ([HV] Definition 5.1). Let $F$ be a facet of a simplicial complex $\Delta$. The connected component of $F$ in $\Delta$, denoted $\operatorname{conn}_{\Delta}(F)$, is defined to be the connected component of $\Delta$ containing $F$. If $\operatorname{conn}_{\Delta}(F)=\left\langle G_{1}, \ldots, G_{p}\right\rangle$, then we define the reduced connected component of $F$ in $\Delta$, denoted by $\overline{\operatorname{conn}}_{\Delta}(F)$, to be the simplicial complex whose facets are a subset of $\left\{G_{1} \backslash F, \ldots, G_{p} \backslash F\right\}$, chosen so that if there exist $G_{i}$ and $G_{j}$ such that $\emptyset \neq G_{i} \backslash F \subseteq G_{j} \backslash F$, then we shall disregard the bigger facet $G_{j} \backslash F$ in $\overline{\operatorname{conn}}_{\Delta}(F)$. 
Note that in the Definition 4.6, $\overline{\operatorname{conn}}_{\Delta}(F)$ is the localization of $\operatorname{conn}_{\Delta}(F)$ at the ideal generated by the complement of the facet $F$. Therefore if $\Delta$ is a tree then $\overline{\operatorname{conn}}_{\Delta}(F)$ is always a forest $([\overline{\mathrm{F} 1}])$. Hà and Van Tuyl ( $[\mathrm{HV}]$ Lemma 5.7) prove this directly in their paper.

A facet $F$ of $\Delta$ is called a splitting facet of $\Delta$ if $\mathcal{F}(\Delta)=(F)+\mathcal{F}(\Delta \backslash\langle F\rangle)$ is a splitting of $\mathcal{F}(\Delta)$ (here we are thinking of $F$ as a monomial).

Theorem 4.7 ([[HV] Theorem 5.5). If $F$ is a splitting facet of a simplicial complex $\Delta$, then for all $i \geqslant 1$ and $j \geqslant 0$ we have

$\beta_{i, j}(\mathcal{F}(\Delta))=\beta_{i, j}(\mathcal{F}(\Delta \backslash\langle F\rangle))+\sum_{l_{1}=0}^{i} \sum_{l_{2}=0}^{j-|F|} \beta_{l_{1}-1, l_{2}}\left(\mathcal{F}\left(\overline{\operatorname{conn}}_{\Delta}(F)\right) \beta_{i-l_{1}-1, j-|F|-l_{2}}\left(\mathcal{F}\left(\Delta \backslash \operatorname{conn}_{\Delta}(F)\right)\right.\right.$.

So now the question is what is a good choice for a splitting facet. In their paper ([山HV Theorem 5.6) Hà and Van Tuyl show that a leaf of a simplicial complex is a splitting facet. Their proof in fact only requires the facet to have a free vertex.

Proposition 4.8. Let $\Delta$ be a simplicial complex. If $F$ is a facet of $\Delta$ with a free vertex, then $F$ is a splitting facet of $\Delta$.

Proof. The proof is identical to the proof of Theorem 5.6 in [HV].

We use the convention that for any ideal $I$

$$
\beta_{-1, j}(I)= \begin{cases}1 & j=0 \\ 0 & \text { otherwise. }\end{cases}
$$

Suppose we have a simplicial tree $\Delta$ with good leaf order described as in Theorem 3.4. We apply (5) to $\Delta=\left\langle F_{0}, \ldots, F_{q}\right\rangle$ peeling off leaves in the following order: $F_{q}, F_{q-1}, \ldots, F_{0}$.

Suppose we are in step $u$, peeling off the leaf $F_{u}$ from the tree $\Delta_{u}=\left\langle F_{0}, \ldots, F_{u}\right\rangle$. Then $\operatorname{conn}_{\Delta_{u}}\left(F_{u}\right)=\Delta_{u}$ and so $\mathcal{F}\left(\Delta_{u} \backslash \operatorname{conn}_{\Delta_{u}}\left(F_{u}\right)\right)=0$ and therefore

$$
\beta_{a, b}\left(\mathcal{F}\left(\Delta_{u} \backslash \operatorname{conn}_{\Delta_{u}}\left(F_{u}\right)\right)= \begin{cases}1 & a=-1, b=0 \\ 0 & \text { otherwise }\end{cases}\right.
$$

Applying this to (5), we solve $i-l_{1}-1=-1$ and $j-\left|F_{u}\right|-l_{2}=0$ to find $l_{1}=i$ and $l_{2}=j-\left|F_{u}\right|$.

Moreover, we have $\overline{\operatorname{conn}}_{\Delta_{u}}\left(F_{u}\right)=\left(\Delta_{u-1}\right)_{\overline{F_{u}}}$, that is $\Delta_{u-1}$ localized at the ideal generated by the complement of the facet $F_{u}$ using notation as in Lemma 2.8. So (5) turns into

$$
\begin{aligned}
\beta_{i, j}(\mathcal{F}(\Delta)) & =\beta_{i, j}\left(\mathcal{F}\left(\Delta_{q-1}\right)\right)+\beta_{i-1, j-\left|F_{q}\right|}\left(\mathcal{F}\left(\left(\Delta_{q-1}\right) \overline{F_{q}}\right)\right) \\
& =\beta_{i, j}\left(\mathcal{F}\left(\Delta_{q-2}\right)\right)+\beta_{i-1, j-\left|F_{q-1}\right|}\left(\mathcal{F}\left(\left(\Delta_{q-2}\right) \overline{F_{q-1}}\right)\right)+\beta_{i-1, j-\left|F_{q}\right|}\left(\mathcal{F}\left(\left(\Delta_{q-1}\right)_{\overline{F_{q}}}\right)\right) \\
& \vdots \\
& =\beta_{i, j}\left(\mathcal{F}\left(\left\langle F_{0}\right\rangle\right)\right)+\sum_{u=1}^{q} \beta_{i-1, j-\left|F_{u}\right|}\left(\mathcal{F}\left(\left(\Delta_{u-1}\right) \overline{F_{u}}\right)\right)
\end{aligned}
$$


Note that we did not use the fact that $F_{0}$ is a good leaf here, just that each $F_{u}$ is a leaf if $\Delta_{u}$. We have therefore justified the following statement.

Proposition 4.9. Let $\Delta$ be a simplicial tree with a good leaf order $F_{0}, F_{1}, \ldots, F_{q}$ such that each $F_{u}$ is a leaf of $\Delta_{u}=\left\langle F_{0}, \ldots, F_{u}\right\rangle$ for $u \leqslant q$. Then for all $i \geqslant 1$ and $j \geqslant 0$

$$
\beta_{i, j}(\mathcal{F}(\Delta))=\beta_{i, j}\left(\mathcal{F}\left(\left\langle F_{0}\right\rangle\right)\right)+\sum_{u=1}^{q} \beta_{i-1, j-\left|F_{u}\right|}\left(\mathcal{F}\left(\left(\Delta_{u-1}\right) \frac{}{F_{u}}\right)\right) .
$$

By introducing an appropriate " $\delta$ " function we can say

$$
\beta_{i, j}\left(\mathcal{F}\left(\left\langle F_{0}\right\rangle\right)\right)=\delta_{(i, j),\left(0,\left|F_{0}\right|\right)}= \begin{cases}1 & i=0, j=\left|F_{0}\right| \\ 0 & \text { otherwise }\end{cases}
$$

So now we focus on the structure of $\left(\Delta_{u-1}\right)_{\overline{F_{u}}}$. The main point that we would like to make is that $\left(\Delta_{u-1}\right)_{\overline{F_{u}}}$ behaves well, in other words, it satisfies the same kind of inclusion sequence enforced in Theorem 3.4 and the same "leaf-peeling" property. Note that though $F_{0}$ need not even survive the localization, its role is that of a virtual glue that forces facets to always stick together and have an appropriate order.

Proposition 4.10. Let $\Delta$ be a simplicial tree with a good leaf $F_{0}$ and good leaf order

$$
F_{0} \cap F_{1} \supsetneq F_{0} \cap F_{2} \supsetneq \cdots \supsetneq F_{0} \cap F_{q} .
$$

Suppose $u \in\{1, \ldots, q\}$ and $\Delta_{u}=\left\langle F_{1}, \ldots, F_{u}\right\rangle$, and suppose $\left(\Delta_{u-1}\right) \overline{F_{u}}$ has facets $F_{a_{1}} \backslash F_{u}, \ldots$, $F_{a_{s}} \backslash F_{u}$ with $0 \leqslant a_{1}<\ldots<a_{s} \leqslant u-1$. Then

1. $a_{s}=u-1$,

2. $\left(F_{0} \cap F_{a_{1}}\right) \backslash F_{u} \supsetneq \ldots \supsetneq\left(F_{0} \cap F_{a_{s}}\right) \backslash F_{u}$,

3. $\left(\Delta_{u-1}\right)_{\overline{F_{u}}}$ is a simplicial tree,

4. If $F_{v}$ is a joint of $F_{u}$ in $\Delta_{u}$ then $F_{v} \backslash F_{u} \in\left(\Delta_{u-1}\right)_{\overline{F_{u}}}$,

5. $F_{u-1} \backslash F_{u}$ has a free vertex in $\left(\Delta_{u-1}\right)_{\overline{F_{u}}}$.

Proof. To prove 1, suppose there is an $i<u-1$ such that $\left(F_{i} \backslash F_{u}\right) \subset\left(F_{u-1} \backslash F_{u}\right)$. By assumption there exists $y \in\left(F_{0} \cap F_{i}\right) \backslash\left(F_{0} \cap F_{u-1}\right)$. As $\left(F_{0} \cap F_{u-1}\right) \supset\left(F_{0} \cap F_{u}\right)$, it follows that $y \in\left(F_{i} \backslash F_{u}\right)$ and $y \notin\left(F_{u-1} \backslash F_{u}\right)$, which contradicts the inclusion $\left(F_{i} \backslash F_{u}\right) \subset\left(F_{u-1} \backslash F_{q}\right)$.

The strict inclusions in 2 follow from the same observation, that for every $i$ there is always an element in $F_{0} \cap F_{a_{i}}$ which is not in $F_{a_{i+1}}$ or $F_{u}$.

Since $\left(\Delta_{u-1}\right)_{\overline{F_{u}}}$ is a localization of the tree $\Delta_{u-1}$, it is clear that it is a forest, and by 2 , since $\left(F_{0} \cap F_{a_{s}}\right) \backslash F_{u} \neq \emptyset$, it must be connected and therefore a simplicial tree. This settles 3 .

For 4, suppose for some $j<u$ we have $F_{j} \backslash F_{u} \subseteq F_{v} \backslash F_{u}$. Then we will have

$$
F_{j}=\left(F_{j} \cap F_{u}\right) \cup\left(F_{j} \backslash F_{u}\right) \subseteq F_{v}
$$


which implies that $F_{j}=F_{v}$.

Finally to prove 5 we use induction on $u$. If $u=1$ or 2 , then $\left(\Delta_{u-1}\right)_{\overline{F_{u}}}$ will have one or two facets, and in each case $F_{u-1} \backslash F_{u}$ clearly must have a free vertex. If $u=3$ then $F_{2}$ is a leaf of $\Delta_{2}$ with a joint $F_{i}$ for some $i<2$. If $F_{i} \backslash F_{3} \in\left(\Delta_{2}\right)_{\overline{F_{3}}}$, then it acts as a joint of $F_{2} \backslash F_{3}$ so $F_{2} \backslash F_{3}$ is a leaf and must therefore have a free vertex. If $F_{i} \backslash F_{3} \notin\left(\Delta_{2}\right)_{\overline{F_{3}}}$, then $\left(\Delta_{2}\right)_{\overline{F_{3}}}$ has at most two facets including $F_{2} \backslash F_{3}$, each of which must have a free vertex. This settles the base cases for induction.

Now suppose $u \geqslant 4$ and $F_{u-1} \backslash F_{u}$ has no free vertex in $\left(\Delta_{u-1}\right)_{\overline{F_{u}}}$.

By induction hypothesis, if we consider $\Gamma=\Delta \backslash\left\langle F_{u-2}\right\rangle$, then $F_{u-1} \backslash F_{u}$ will have a free vertex $x$ in $\left(\Gamma_{u-1}\right)_{\overline{F_{u}}}$. If $x$ is not a free vertex in $\left(\Delta_{u-1}\right) \overline{F_{u}}$, then for some $j<u-1$ we have $x \in F_{j} \backslash F_{u} \in\left(\Delta_{u-1}\right) \overline{F_{u}}$ and $F_{j} \backslash F_{u} \notin\left(\Gamma_{u-1}\right) \overline{F_{u}}$. The only possible such index $j$ is $j=u-2$. In other words, $x \in F_{u-1} \cap F_{u-2}$ and $x \notin F_{i}$ for any other $i \leqslant u$.

Similarly, if we remove $F_{u-3}$ from $\Delta$ we will find a vertex $y \in F_{u-1} \cap F_{u-3}$ and $y \notin F_{i}$ for any other $i \leqslant u$.

By Lemma 3.2. we must then have $F_{u-3} \cap F_{u-2} \subseteq F_{u-1}$. Intersecting both sides with $F_{0}$ we obtain

$$
F_{u-1} \cap F_{0} \subseteq F_{u-2} \cap F_{0}=F_{u-3} \cap F_{u-2} \cap F_{0} \subseteq F_{u-1} \cap F_{0}
$$

which means that $F_{u-1} \cap F_{0}=F_{u-2} \cap F_{0}$; a contradiction.

Proposition 4.10 now allows us to continue solving (7) by applying Theorem 4.7 once again, since we have a splitting facet for each $\left(\Delta_{u-1}\right)_{\overline{F_{u}}}$. Consider the tree $\Delta$ as described above with the good leaf order described in Theorem 3.4 and for some $u \in\{1, \ldots, q\}$, let $\left(\Delta_{u-1}\right)_{\overline{F_{u}}}=\left\langle F_{a_{1}}\right\rangle$ $\left.F_{u}, \ldots, F_{a_{s}} \backslash F_{u}\right\rangle$ where $0 \leqslant a_{1}<\ldots<a_{s}<u$. By Proposition $4.10\left(\Delta_{u-1}\right)_{\overline{F_{u}}}$ is a simplicial tree with an order of the facets induced by the good leaf order of $\Delta$, and with splitting facet $F_{a_{s}} \backslash F_{u}$.

We continue in the same spirit. Let $u_{1}=u, u_{2}=a_{s}$ and

$$
\mathcal{C}_{u_{1}, u_{2}}=\left(\left(\Delta_{u_{1}-1}\right)_{F_{u_{1}}}\right)_{F_{u_{2}} \backslash F_{u_{1}}}=\left\langle F_{d_{1}} \backslash\left(F_{u_{1}} \cup F_{u_{2}}\right), \ldots, F_{d_{w}} \backslash\left(F_{u_{1}} \cup F_{u_{2}}\right)\right\rangle
$$

where $0 \leqslant d_{1}<\ldots<d_{w}<u_{2}<u_{1}$.

Similarly, we can build $\mathcal{C}_{u_{1}, \ldots, u_{m}}$ which is the localization of

$$
C_{u_{1}, \ldots, u_{m-1}}=\left\langle F_{c_{1}} \backslash\left(F_{u_{1}} \cup \ldots \cup F_{u_{m-1}}\right), \ldots, F_{c_{r}} \backslash\left(F_{u_{1}} \cup \ldots \cup F_{u_{m-1}}\right)\right\rangle
$$

at the ideal generated by the complement of the facet $F_{u_{m}} \backslash\left(F_{u_{1}} \cup \ldots \cup F_{u_{m-1}}\right)$ where $u_{m}=c_{r}$. So we have

$$
\mathcal{C}_{u_{1}, \ldots, u_{m}}=\left\langle F_{b_{1}} \backslash\left(F_{u_{1}} \cup \ldots \cup F_{u_{m}}\right), \ldots, F_{b_{t}} \backslash\left(F_{u_{1}} \cup \ldots \cup F_{u_{m}}\right)\right\rangle
$$

where $b_{1}, \ldots, b_{t} \in\left\{c_{1}, \ldots, c_{r-1}\right\}$, and

$$
0 \leqslant b_{1}<b_{2}<\ldots<b_{t}<c_{r}=u_{m}<u_{m-1}<\ldots<u_{1}
$$

Proposition 4.11. Let $\Delta$ be a simplicial tree with a good leaf $F_{0}$ and good leaf order

$$
F_{0} \cap F_{1} \supsetneq F_{0} \cap F_{2} \supsetneq \cdots \supsetneq F_{0} \cap F_{q} .
$$

With notation as in (9) and (10) above, we have 
1. $b_{t}=c_{r-1}$,

2. $\left(F_{0} \cap F_{b_{1}}\right) \backslash\left(F_{u_{1}} \cup \ldots \cup F_{u_{m}}\right) \supsetneq \ldots \supsetneq\left(F_{0} \cap F_{b_{t}}\right) \backslash\left(F_{u_{1}} \cup \ldots \cup F_{u_{m}}\right)$,

3. $\mathcal{C}_{u_{1}, \ldots, u_{m}}$ is a simplicial tree,

4. $F_{b_{t}} \backslash\left(F_{u_{1}} \cup \ldots \cup F_{u_{m}}\right)$ has a free vertex in $\mathcal{C}_{u_{1}, \ldots, u_{m}}$ and is therefore a splitting facet of $\mathcal{C}_{u_{1}, \ldots, u_{m}}$.

Proof. Let $A=F_{u_{1}} \cup \ldots \cup F_{u_{m}}$. To show 1, suppose there is an $i<r-1$ such that $F_{c_{i}} \backslash A \subset$ $F_{c_{r-1}} \backslash A$. By the strict inclusions assumed there exists $y \in\left(F_{0} \cap F_{c_{i}}\right) \backslash\left(F_{0} \cap F_{c_{r-1}}\right)$. As

$$
F_{0} \cap F_{c_{r-1}} \supsetneq F_{0} \cap F_{u_{m}} \supsetneq \ldots \supsetneq F_{0} \cap F_{u_{1}},
$$

it follows that $y \in F_{c_{i}} \backslash A$ and $y \notin F_{c_{r-1}} \backslash A$, which is a contradiction.

For 2 it is easy to see that

$$
\left(F_{0} \cap F_{b_{1}}\right) \backslash A \supseteq \ldots \supseteq\left(F_{0} \cap F_{b_{t}}\right) \backslash A .
$$

To show that these inclusions are strict pick $1 \leqslant i<j<t$, we know that

$$
F_{0} \cap F_{b_{i}} \supsetneq F_{0} \cap F_{b_{j}} \supsetneq F_{0} \cap F_{u_{m}} \supsetneq F_{0} \cap F_{u_{m-1}} \supsetneq \ldots \supsetneq F_{0} \cap F_{u_{1}},
$$

and therefore there exists $y \in\left(F_{b_{i}} \cap F_{0}\right) \backslash\left(F_{b_{j}} \cup F_{u_{1}} \cup \ldots \cup F_{u_{m}}\right)$, which means that $y \in$ $\left(F_{0} \cap F_{b_{i}}\right) \backslash\left(F_{u_{1}} \cup \ldots \cup F_{u_{m}}\right)$ and $y \notin\left(F_{0} \cap F_{b_{j}}\right) \backslash\left(F_{u_{1}} \cup \ldots \cup F_{u_{m}}\right)$, proving 2 .

Suppose $\Omega=\left\langle F_{\omega_{0}}, F_{\omega_{1}}, \ldots, F_{\omega_{p}}\right\rangle$ is the subcollection of $\Delta$ consisting of those facets that are not contained in $A$ with

$$
0=\omega_{0}<\omega_{1}<\ldots<\omega_{p} .
$$

Because of the strict good leaf order $\Omega$ is a connected forest and hence a tree.

We claim that $C_{u_{1}, \ldots, u_{m}}$ is the localization of the tree $\Omega$ at the ideal generated by $\bar{A}$. This follows from two observations. One is that if at the $i$ th step when building $C_{u_{1}, \ldots, u_{m}}$ there are facets $F_{\alpha}, F_{\beta} \in \Delta$ not containing $F_{u_{1}} \cup \ldots \cup F_{u_{i}}$, then $F_{\alpha}, F_{\beta}$ do not contain $A$ and therefore are also facets of $\Omega$. Moreover if $F_{\alpha} \backslash\left(F_{u_{1}} \cup \ldots \cup F_{u_{i}}\right) \subseteq F_{\beta} \backslash\left(F_{u_{1}} \cup \ldots \cup F_{u_{i}}\right)$, then $F_{\alpha} \backslash A \subseteq F_{\beta} \backslash A$ and therefore we can conclude that $C_{u_{1}, \ldots, u_{m}}$ is a localization $\Omega$ and $\left\{b_{1} \ldots b_{t}\right\} \subseteq\left\{\omega_{0}, \ldots, \omega_{p}\right\}$.

So $C_{u_{1}, \ldots, u_{m}}$ must be a forest, and since it is connected by 2 , it must be a simplicial tree. This settles 3.

By the discussion above we can assume $\omega_{p}=b_{t}$ and we will still have $C_{u_{1}, \ldots, u_{m}}$ is a localization of $\Omega$. Also note that $F_{0}=F_{\omega_{0}}$ is a good leaf of $\Omega$ with a strict good leaf order induced by that on $\Delta$.

To prove 4 we use induction on $p$. If $p=1$ or 2 then $C_{u_{1}, \ldots, u_{m}}$ will have one or two facets, and in each case $F_{b_{t}} \backslash A$ clearly must have a free vertex. If $p=3$ then $F_{\omega_{2}}$ is a leaf of $\Omega_{\omega_{2}}=$ $\left\langle F_{\omega_{0}}, F_{\omega_{1}}, F_{\omega_{2}}\right\rangle$ with a joint $F_{w_{i}}$ for some $i<2$. If $F_{\omega_{i}} \backslash A \in C_{u_{1}, \ldots, u_{m}}$, then it acts as a joint of $F_{\omega_{2}} \backslash A$ so $F_{\omega_{2}} \backslash A$ is a leaf and must therefore have a free vertex. If $F_{\omega_{i}} \backslash A \notin C_{u_{1}, \ldots, u_{m}}$, then $C_{u_{1}, \ldots, u_{m}}$ has at most two facets including $F_{\omega_{2}} \backslash A$ each of which must have a free vertex. This settles the base cases for induction. 
Now suppose $p \geqslant 4$ and $F_{b_{t}} \backslash A$ has no free vertex in $C_{u_{1}, \ldots, u_{m}}$.

By the induction hypothesis, if we consider $\Gamma=\Omega \backslash\left\langle F_{\omega_{p-1}}\right\rangle$ then $F_{\omega_{p}}$ will have a free vertex $x$ in $\Gamma_{\bar{A}}$. If $x$ is not a free vertex in $\Gamma_{\bar{A}}$ then $x \in F_{\omega_{p-1}} \backslash A \in \Gamma_{\bar{A}}$. In other words, $x \in F_{\omega_{p}} \cap F_{\omega_{p-1}}$ and $x \notin F_{\omega_{i}}$ for any other $i \leqslant p$.

Similarly, if we remove $F_{\omega_{p-2}}$ from $\Omega$ we will find a vertex $y \in F_{\omega_{p}} \cap F_{\omega_{p-2}}$ and $y \notin F_{i}$ for any other $i \leqslant p$.

By Lemma 3.2, we must then have $F_{\omega_{p-2}} \cap F_{\omega_{p-1}} \subseteq F_{\omega_{p}}$. Intersecting both sides with $F_{0}$ we obtain

$$
F_{\omega_{p}} \cap F_{0} \subseteq F_{\omega_{p-1}} \cap F_{0}=F_{\omega_{p-2}} \cap F_{\omega_{p-1}} \cap F_{0} \subseteq F_{\omega_{p}} \cap F_{0}
$$

which means that $F_{\omega_{p}} \cap F_{0}=F_{\omega_{p-1}} \cap F_{0}$; a contradiction. This proves 4 and we are done.

Proposition 4.11 replaces Proposition 4.10 as a more general version. Back to (7), we start computing Betti numbers of $\mathcal{F}(\Delta)$ for a given tree $\Delta$ with good leaf $F_{0}$ and strict good leaf order

$$
F_{0} \cap F_{1} \supsetneq F_{0} \cap F_{2} \supsetneq \cdots \supsetneq F_{0} \cap F_{q} .
$$

The formula

$$
\beta_{i, j}(\mathcal{F}(\Delta))=\beta_{i, j}\left(\mathcal{F}\left(\left\langle F_{0}\right\rangle\right)\right)+\sum_{u=1}^{q} \beta_{i-1, j-\left|F_{u}\right|}\left(\mathcal{F}\left(\left(\Delta_{u-1}\right)_{\overline{F_{u}}}\right)\right)
$$

becomes recursive, since in each step after localization we again have a simplicial tree with a strict induced order on the facets where the last facet remaining is a splitting facet.

To close, we apply the formula to examine some low Betti numbers.

Let $i=0$. By (7) and (6) we have

$$
\beta_{0, j}(\mathcal{F}(\Delta))=\sum_{u=0}^{q} \delta_{j,\left|F_{u}\right|}
$$

Let $i \geqslant 1$. Because of (7) and (8) we can write

$$
\beta_{i, j}(\mathcal{F}(\Delta))=\sum_{u=1}^{q} \beta_{i-1, j-\left|F_{u}\right|}\left(\mathcal{F}\left(\left(\Delta_{u-1}\right)_{\overline{F_{u}}}\right)\right)
$$

From Proposition 4.11 and (11) we can see that we need the generators of each $\Delta_{u}$ in order to produce a formula for the first graded Betti numbers. To this end, we start from $\Delta_{u}=\left\langle F_{0}, \ldots, F_{u}\right\rangle$ so that

$$
\begin{aligned}
\left(\Delta_{u-1}\right)_{\overline{F_{u}}} & \left.=\left\langle F_{i} \backslash F_{u}\right| 0 \leqslant i<u \text { and }\left(F_{j} \backslash F_{u}\right) \not \subset\left(F_{i} \backslash F_{u}\right) \text { for } j \neq i\right\rangle \\
& \left.=\left\langle F_{i} \backslash F_{u}\right| 0 \leqslant i<u \text { and } \frac{\operatorname{lcm}\left(F_{j}, F_{u}\right)}{F_{u}} \nmid \frac{\operatorname{lcm}\left(F_{i}, F_{u}\right)}{F_{u}} \text { for } j \neq i\right\rangle \\
& \left.=\left\langle F_{i} \backslash F_{u}\right| 0 \leqslant i<u \text { and } \operatorname{lcm}\left(F_{j}, F_{u}\right) \backslash \operatorname{lcm}\left(F_{i}, F_{u}\right) \text { for } j \neq i\right\rangle
\end{aligned}
$$


So we can make our "delta-function" to have the lcm condition built into it. We define

$$
\delta_{a,(b, c)}= \begin{cases}1 & a=\left|F_{b}\right|, \operatorname{lcm}\left(F_{d}, F_{c}\right) \backslash \operatorname{lcm}\left(F_{b}, F_{c}\right) \text { for } 0 \leqslant d<c \\ 0 & \text { otherwise }\end{cases}
$$

So (11) becomes

$$
\begin{aligned}
\beta_{1, j}(\mathcal{F}(\Delta)) & =\sum_{u=1}^{q} \beta_{0, j-\left|F_{u}\right|}\left(\mathcal{F}\left(\left(\Delta_{u-1}\right)_{\overline{F_{u}}}\right)\right) \\
& =\sum_{u=1}^{q} \sum_{F \text { facet of }\left(\Delta_{u-1}\right)_{\overline{F_{u}}}} \delta_{j-\left|F_{u}\right|,|F|} \\
& =\sum_{u=1}^{q} \sum_{v=0}^{u-1} \delta_{j-\left|F_{u}\right|,(v, u)}
\end{aligned}
$$

By building appropriate delta functions, one can continue in this manner to build further Betti numbers based on the lcms of the facets.

\section{References}

[CFS] M. Caboara, S. Faridi, P. Selinger, Simplicial cycles and the computation of simplicial trees, J. Symbolic Comput. 42, no. 1-2, 74-88 (2007).

[EK] S. Eliahou and M. Kervaire, Minimal resolutions of some monomial ideals, J. Algebra 129, no. 1, 1-25 (1990).

[F1] S. Faridi, The facet ideal of a simplicial complex, Manuscripta Mathematica 109, 159-174 (2002).

[F2] S. Faridi, Cohen-Macaulay properties of square-free monomial ideals, Journal of Combinatorial Theory, Series A, 109, no. 2, 299-329 (2005).

[Fa] G. Fatabbi, On the resolution of ideals of fat points, J. Algebra 242, no. 1, 92-108 (2001).

[Fr] R. Fröberg, A study of graded extremal rings and of monomial rings, Math. Scand. 51, 22-34 (1982).

[M2] D. R. Grayson, M. E. Stillman, Macaulay2, a software system for research in algebraic geometry, Available at http://www.math.uiuc.edu/Macaulay2/.

[GPW] V. Gasharov, I. Peeva, V. Welker, The lcm-lattice in monomial resolutions, Math. Res. Lett. 6, no. 5-6, 521-532 (1999).

[HHTZ] J. Herzog, T. Hibi, N.V. Trung, X. Zheng, Standard graded vertex cover algebras, cycles and leaves, Trans. Amer. Math. Soc. 360, no. 12, 6231-6249 (2008). 
[HV] H. T. Hà and A. Van Tuyl, Splittable ideals and the resolutions of monomial ideals, Journal of Algebra 309, 405-425 (2007).

[V] R. Villarreal, Monomial algebras, Monographs and Textbooks in Pure and Applied Mathematics, 238. Marcel Dekker, Inc., New York (2001).

[Z] X. Zheng, Homological properties of monomial ideals associated to quasi-trees and lattices, Ph.D. thesis, Universität Duisburg-Essen (August 2004). 